\title{
Current status of lung transplantation in Hong Kong
}

\author{
See Wan Yan ${ }^{1}$, Chi Fong Wong ${ }^{1}$, Kuan Yew Michael Hsin ${ }^{2}$, Ka Lai Cally Ho ${ }^{2}$, Ko Yung Sit ${ }^{2}$, \\ Wing Kuk Timmy $\mathrm{Au}^{2}$
}

${ }^{1}$ Tuberculosis and Chest Unit, Grantham Hospital, Hong Kong, China; '2Department of Cardiothoracic Surgery, Queen Mary Hospital, Hong Kong, China

Contributions: (I) Conception and design: SW Yan, CF Wong; (II) Administrative support: SW Yan, CF Wong; (III) Provision of study materials or patients: All authors; (IV) Collection and assembly of data: SW Yan; (V) Data analysis and interpretation: SW Yan, CF Wong; (VI) Manuscript writing: All authors; (VII) Final approval of manuscript: All authors.

Correspondence to: Dr. See Wan Yan. Tuberculosis and Chest Unit, Grantham Hospital, 5/F TBCU Office, 125 Wong Chuk Hang Road, Aberdeen, Hong Kong, China. Email: yansw2@ha.org.hk.

\begin{abstract}
The only lung transplant program in Hong Kong, which is jointly led by respiratory physicians in Tuberculosis and Chest Unit of Grantham Hospital (GH) and surgeons in Cardiothoracic Surgical Department of Queen Mary Hospital (QMH), has been running for 25 years. The programme was running initially at low profile and with a low case volume in the first decade. The case load has increased significantly in the last decade. Among 187 potential recipients that had been put on waiting list so far, 77 had received lung transplants and 70 died while on waiting list. Majority of the lung transplant surgeries were bilateral sequential lung transplant. Common underlying diagnoses for lung transplant in Hong Kong included chronic obstructive pulmonary disease (COPD), suppurative lung diseases, pulmonary vascular disease, post haemopoietic stem cell transplant bronchiolitis obliterans syndrome (BOS), interstitial lung diseases (ILDs), and lymphangioleiomyomatosis (LAM). The mean age of recipients at transplant was 47.26 years. The 1-, 3-, 5- and 10-year survival were $84.4 \%, 74.9 \%, 64.1 \%$ and $50.3 \%$ respectively. The rate of acute rejection and chronic lung allograft dysfunction (CLAD) were low compared to international data. With a very low organ donation rate (about 6 per-million-population yearly) in the territory, shortage of donor organs remains the biggest unmet need for lung transplantation in Hong Kong.
\end{abstract}

Keywords: Lung transplantation; Hong Kong; survival

Received: 29 May 2020; Accepted: 25 September 2020; Published: 25 February 2022.

doi: $10.21037 /$ ccts-20-109

View this article at: http://dx.doi.org/10.21037/ccts-20-109

\section{Introduction}

Since the first successful case in early 1980's, lung transplantation has evolved into a well-accepted treatment option for selected patients with advanced pulmonary diseases. According to the registry data from the International Society for Heart and Lung Transplantation (ISHLT), there are currently over four thousand lung transplants being performed each year in over a hundred transplant centers worldwide (1). The majority of the lung transplant centers are located in Europe and North America and they are responsible for over $90 \%$ of the cases.

\section{Lung transplantation in Hong Kong: history and setting}

Like many western countries, organ transplant in Hong Kong is regulated under the Ordinance which, inter alia, prohibits commercial dealings in human organs intended for transplanting and restricts the transplanting of imported organs. Currently, all cadaveric organ transplants are from brain-death donors. The cadaveric organ donation and transplant are coordinated under the Hospital Authority, which is a government funded public body responsible for all public hospital medical services. An opt-in system is 
adopted based on voluntary decisions of people to donate and the family consent to the donation after brain death for cadaveric organ donation. Donation after circulatory death is currently not practiced in Hong Kong.

The only lung transplant program in Hong Kong was launched in 1994 in Grantham Hospital (GH), jointly run by respiratory physicians in Tuberculosis and Chest Unit and surgeons in Cardiothoracic Surgical Department. The first case of lung transplantation (single lung) was performed in July 1995 for a young lady suffering from lymphangioleiomyomatosis (LAM). The first heart-lung transplant was performed in December 1995 and the first bilateral lung transplant was performed in 1997. Since 2008, with the relocation of the Cardiothoracic Surgical Department to Queen Mary Hospital (QMH), a universityaffiliated general hospital nearby, the transplant program remained largely unchanged except the transplant surgery and the early post-operative care being carried out in QMH. Currently, only cadaveric lung transplants are being performed in Hong Kong.

\section{Referrals and evaluation of potential recipients}

In Hong Kong, the lung transplant program received all potential patients with end stage respiratory failure for assessment. The selection criteria for potential lung transplant recipients were largely based on the guidelines published by the ISHLT (2), and the upper age limit of the referral was set at 65 years. All potential recipients underwent an extensive work up including blood tests, physiological assessment, imaging studies and cardiac assessment. The decision for acceptance for transplant waitlisting was jointly made by the transplant physicians and surgeons.

\section{Donor assessment and lung transplant surgery}

Donor assessment was done by respiratory physicians including on-site fibre-optic bronchoscopy examination. Lung organ harvest was carried out as part of the multiorgan harvesting surgery in the standard manner by cardiothoracic surgeons.

Most of the lung transplant surgeries in Hong Kong were bilateral sequential lung transplant under general anesthesia. Intra-operative extracorporeal membraneous oxygenation (ECMO) was generally preferred over cardiopulmonary bypass except in situations where concomitant cardiac procedures (for instance, tricuspid annuloplasty or closure of atrial septal defect) were needed or when full intraoperative circulatory support was required for left or right heart dysfunction or severe bleeding was encountered (3). Both transverse thoraco-sternotomy (clamshell) approach and bilateral thoracotomy approach were used as per cardiothoracic surgeons' preference. Post lung-transplant patients were managed in the Cardiothoracic Intensive Care Unit in QMH. After initial stabilization, patients were transferred to $\mathrm{GH}$ to continue care including rehabilitation before discharge. All patients were followed up in GH life-long for post-transplant care and monitoring, including regular surveillance bronchoscopic examinations with transbronchial lung biopsies at 1 month, 3 months, 6 months and 1 year.

\section{Immunosuppression protocol}

The immunosuppression protocol for lung transplant was similar to those in most other centers. Basilizumab is currently the induction agent being employed and given at perfusion of lung graft. Intravenous methylprednisolone was given immediately prior to transplant and before perfusion of the graft. Mycophenolate mofetil was also being administered before transplant. For maintenance immunosuppression, a triple immunosuppression regimen using tacrolimus, mycophenolate mofetil and prednisolone was generally adopted.

\section{Antimicrobial prophylaxis}

Routine anti-microbial prophylaxis to cover bacteria, fungus and cytomegalovirus (CMV) were given.

Antibiotic regimen mostly included cefepime or agents targeted against the known previous positive respiratory isolates. The duration depended on the subsequent clinical condition and corresponding respiratory culture results. Co-trimoxazole was given life-long for prophylaxis against pneumocystic jeroveci. Anti-fungal cover included initial intravenous echinocandin (anidulafungin or micafungin) for 2 weeks and subsequent oral azole (mostly itraconazole) for a year as routine. CMV prophylaxis in the form of intravenous ganciclovir followed by oral valganciclovir was intended for 3 months after transplant. Acyclovir would be given after oral valganciclovir for patients at risk of herpes infections. One year of anti-tuberculous prophylaxis using isoniazid was given to patients with prior history of tuberculosis or a positive tuberculin skin test before transplant. 


\section{Changes in case volume over time}

The lung transplant program in Hong Kong had been running at a very small scale and low profile in the first decade from 1990s to 2000s. An average of just about one lung transplant was being performed each year. It was largely due to limited resources and adoption of a more conservative approach in line with the contemporary view in global transplantation field. With accumulation of experience and advancement of technical skills, alongside the effort in promotion of organ donation and transplantation in the local community, the number of lung transplants had increased significantly since 2010 s. Over a 5-year period from 2015 to 2019, the number of lung transplants being performed was 49 , which has nearly doubled the number of cases in the preceding 20 years from 1995 to 2014 (Figure 1).

\section{Recipients' characteristics and disease pattern}

Since the launch of the lung transplant program in Hong Kong, there has been 187 patients being put on waiting list after evaluation. Seventy (37.4\%) patients had passed away while waiting for lung transplant (Figure 2). The high wait-list mortality was mainly due to the scarcity

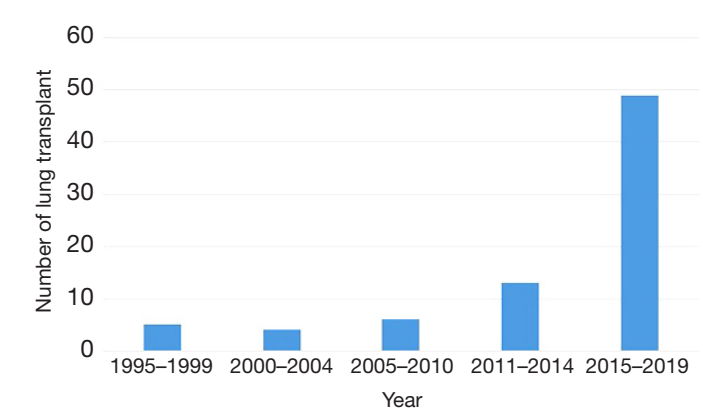

Figure 1 Growing trend of lung transplantation in Hong Kong. of lung organ donors. Like the situation in many other countries, low organ donation rate was a great hurdle to organ transplantation. The local donation rate of about six cadaveric organ donations per-million-population was among the lowest worldwide. The average waiting time for a lung transplant was as long as 16.7 months in 2019. According to a local survey in 2019 , the main reason $(72.5 \%)$ for not willing to donate their organs after death was reported as "would like to keep their body intact", which was a common cultural belief in Chinese (4).

Seventy-seven patients had received lung transplants (6 single and 71 double) in Hong Kong from 1995 to 2019. Male to female ratio approximated equal preponderance (40:37). The age at transplant ranged from 21 to 63 years and the mean was 49.26 years. Although there had been pediatric patients being put on lung transplant waiting list, no pediatric lung transplant had been performed in Hong Kong.

The disease categories of lung transplant cases in Hong Kong included 24 (31.2\%) chronic obstructive pulmonary disease (COPD), 15 (19.5\%) suppurative lung disease, 12 (15.6\%) pulmonary vascular disease, 7 (9.1\%) post bone marrow transplant bronchiolitis obliterans syndrome (post BMT BOS), 8 (10.4\%) interstitial lung disease (ILD), 8 (10.4\%) LAM and 3 (3.9\%) others (Figure 3). This pattern differs markedly from the worldwide statistics reported by ISHLT (1), in which ILD [including both idiopathic interstitial pneumonia (IIP) and non-IIP ILD] accounted for $31.8 \%$, and COPD accounted for $30.1 \%$ of lung transplant cases from 1995 to 2018. Because of the absence of those genetic abnormalities in southern Chinese population, there were no cystic fibrosis and alpha-1-anti-trypsin deficiency cases seen in Hong Kong. The higher representation of pulmonary vascular disease, post BMT BOS and LAM locally may be related to their more indolent disease course and these patients could stay alive in the waiting list for longer period of time.

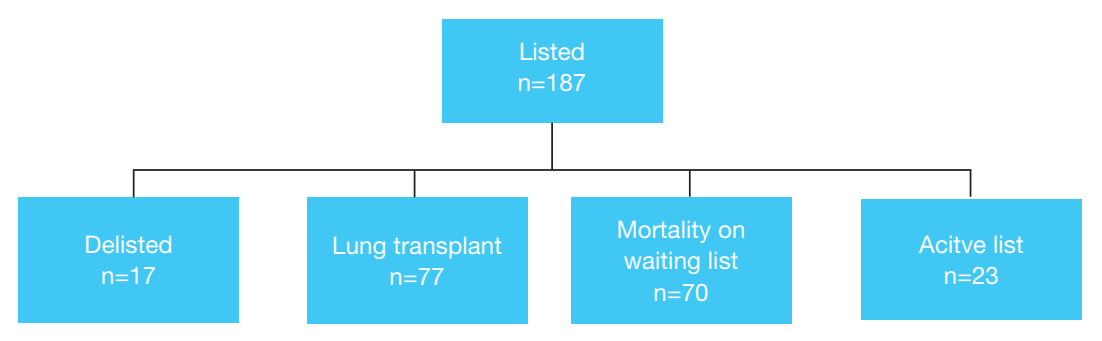

Figure 2 Clinical course of patients on waiting list. 


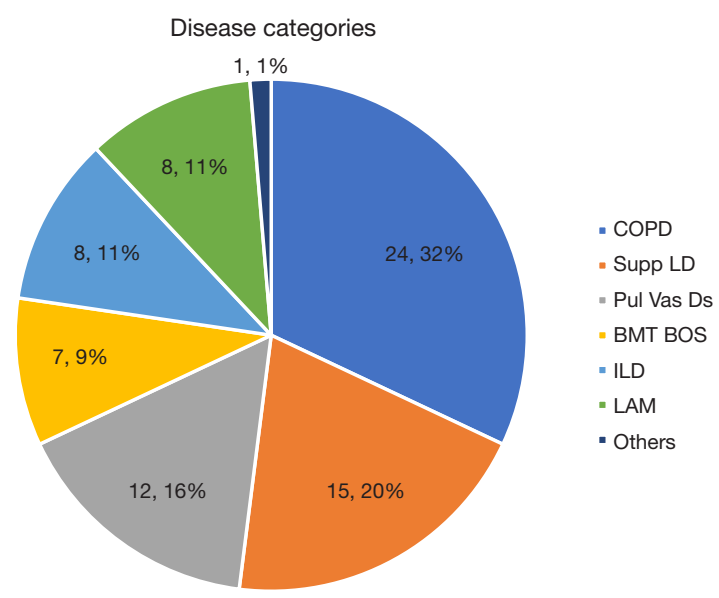

Figure 3 Lung transplantation by disease categories. COPD, chronic obstructive pulmonary disease; ILD, interstitial lung disease; BOS, bronchiolitis obliterans syndrome; LAM, lymphangioleiomyomatosis.

Table 1 Survival rate of lung transplant patients

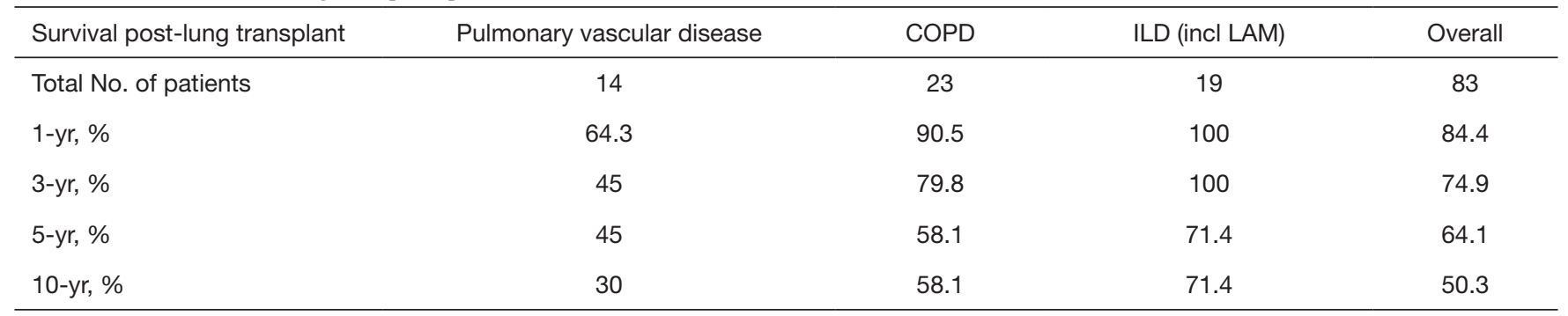

COPD, chronic obstructive pulmonary disease; ILD, interstitial lung disease; LAM, lymphangioleiomyomatosis.

\section{Survival}

Our lung transplant program has favorable results in terms of survival albeit its small size when compared to the ISHLT registry database. The overall 1-, 3-, 5- and 10year survival rate were $84.4 \%, 74.9 \%, 64.1 \%$ and $50.3 \%$ respectively at the end of 2019. The survival rate was best for patients with ILDs. The worst survival outcome belonged to patients with pulmonary vascular disease (Table 1). Our patient with the longest survival is a female patient with LAM who had a single lung transplant 23 years ago. She is currently enjoying reasonably independent lifestyle and is still working full time.

\section{Acute rejection and chronic lung allograft dysfunction (CLAD)}

Upon review of 59 patients who underwent lung transplant in the past 10 years from 2010 to 2019 , there were $6(10.2 \%)$ patients-episodes of acute rejection requiring intensive treatment. Eleven patients $(18.6 \%)$ had developed CLAD and among which 8 ended up in mortality. The data compared favorably with the international benchmarks from ISHLT data in 2019 which included patients from 2005 to 2018 in that $26.6 \%$ of patients experienced at least one episode of treated acute rejection within the first year of transplant and the annual incidence of BOS was quoted as $10 \%$ of patients per year (1).

\section{Infection episodes and malignancy}

Fungal infection, mostly aspergillus is a significant threat to patients in early post lung transplant period. In the 10 years' period from 2010 to 2019 , there were twelve fungal infection episodes in our 59 post lung transplant patients. The fungal pathogens were Aspergillus [8], Candida [2], Cryptococcus [1] and trichosporon [1]. There were four episodes of CMV infection. There was also high incidence 
of tuberculosis [2] and non-tuberculosis mycobacterial infection [6] in our cohort of patients. It could be well explained by the high local prevalence as an endemic area in the world for mycobacterial diseases.

Malignancy was a rare occurrence from 2010 to 2019 with only one patient developed carcinoma of colon 1 year after lung transplant.

\section{Challenges and way forward}

Hong Kong remains a relatively small volume center for lung transplantation though an increasing trend of activity is observed in recent years. However, the long waiting time and hence the high wait-list mortality are still the major unresolved issues. The scarcity of donors locally, like other transplant centers, is a global concern in lung transplant field. In recent years, the use of ex-vivo lung perfusion techniques for borderline donor lung organs and lung organ donation after circulatory death have received much attention with encouraging results reported in some parts of the world to relieve the unmet needs of lung transplant due to scarcity of organ donors.

\section{Acknowledgments}

We acknowledge all staff's work in the lung transplant team including pulmonologists, cardiologists, pathologists, physiotherapists, occupational therapists, lung function laboratory technologists, dietitians, clinical psychologists, nurses, supporting and clerical staff who contributed to the manuscript, but who do not qualify for inclusion as authors. Funding: None.

\section{Footnote}

Provenance and Peer Review: This article was commissioned by the editorial office, Current Challenges in Thoracic Surgery for the series "Highlights of the First International Symposium on Lung transplantation, Wuxi, China, 2019". The article has undergone external peer review.

Peer Review File: Available at https://ccts.amegroups.com/ article/view/10.21037/ccts-20-109/prf

Conflicts of Interest: All authors have completed the ICMJE uniform disclosure form (available at https://ccts.amegroups. com/article/view/10.21037/ccts-20-109/coif). The series
"Highlights of the First International Symposium on Lung transplantation, Wuxi, China, 2019" was commissioned by the editorial office without any funding or sponsorship. KYMH served as the unpaid Guest Editor of the series. The authors have no other conflicts of interest to declare.

Ethical Statement: The authors are accountable for all aspects of the work in ensuring that questions related to the accuracy or integrity of any part of the work are appropriately investigated and resolved.

Open Access Statement: This is an Open Access article distributed in accordance with the Creative Commons Attribution-NonCommercial-NoDerivs 4.0 International License (CC BY-NC-ND 4.0), which permits the noncommercial replication and distribution of the article with the strict proviso that no changes or edits are made and the original work is properly cited (including links to both the formal publication through the relevant DOI and the license). See: https://creativecommons.org/licenses/by-nc-nd/4.0/.

\section{References}

1. Chambers DC, Cherikh WS, Harhay MO, et al. The International Thoracic Organ Transplant Registry of the International Society for Heart and Lung Transplantation: Thirty-sixth adult lung and heart-lung transplantation Report-2019; Focus theme: Donor and recipient size match. J Heart Lung Transplant 2019;38:1042-55.

2. Weill D, Benden C, Corris PA, et al. A consensus document for the selection of lung transplant candidates: 2014--an update from the Pulmonary Transplantation Council of the International Society for Heart and Lung Transplantation. J Heart Lung Transplant 2015;34:1-15.

3. McFadden PM, Greene CL. The evolution of intraoperative support in lung transplantation: cardiopulmonary bypass to extracorporeal membrane oxygenation. J Thorac Cardiovasc Surg 2015;149:1158-60.

4. Census and Statistic Department, HKSAR. Thematic household survey report No. 68. 2019.

doi: $10.21037 /$ ccts-20-109

Cite this article as: Yan SW, Wong CF, Hsin KYM, Ho KLC, Sit KY, Au WKT. Current status of lung transplantation in Hong Kong. Curr Chall Thorac Surg 2022;4:3. 